\title{
Current Issues in the Diagnosis and Management of Blood-Culture Negative Infective and Non-Infective Endocarditis
}

\author{
Anthi Katsouli, MD \\ Malek G. Massad, MD, FACS \\ Division of Cardiothoracic Surgery; University of Illinois at Chicago, Chicago, \\ Illinois. \\ Running Heading: Culture Negative Endocarditis
}

Word count: 6218

Address for Correspondence:

Malek G. Massad, MD, FACS

Division of Cardiothoracic Surgery

University of Illinois at Chicago

840 S. Wood Street, CSB Suite 417 (MC 958)

Chicago, Illinois 60612

e-mail: mmassad@uic.edu 


\begin{abstract}
Diagnosis and management of blood culture negative endocarditis constitute a formidable clinical challenge and a systemic approach is necessary for a successful outcome. Blood cultures are negative in endocarditis due mainly to preceding antibiotic administration or to fastidious slow-growing organisms. Less so, non-infective endocarditis as a paraneoplastic manifestation or may occur in association with autoimmune diseases. When the clinical diagnosis is contemplated and cultures and serologies are negative, histologic and molecular examination of the removed valve tissue may confirm the diagnosis. Treatment with antibiotics is often warranted and valve replacement remains appropriate for patients with heart failure or irreversible structural damage.
\end{abstract}

Key words: Blood culture-negative endocarditis; Infective and non-infective endocarditis; Nonbacterial thrombotic endocarditis; Diagnosis and management. 


\section{Introduction}

Blood-culture negative endocarditis (BCNE) refers to definite or probable endocarditis in which three or more aerobic and anaerobic blood cultures collected over 48 hours remain negative despite prolonged (>1 week) incubation $[1,2]$. It constitutes a challenge to the practicing physician and is often associated with higher morbidity and mortality compared with blood culture positive endocarditis (BCPE).

The goals of the present review are to provide an up-to-date current knowledge on infective and noninfective BCNE and to suggest strategies for the diagnosis and management of this condition.

\section{Epidemiology and Risk Factors}

BCNE accounts for 2.5-31\% of all cases of endocarditis [3]. This variation may be explained by differences in the diagnostic criteria and sampling strategies used; specific epidemiological factors affecting some fastidious zoonotic agents; variations in the early use of antibiotics prior to blood sampling; and involvement of unknown pathogens (Figure 1).

Risk factors for culture negative infective endocarditis include the risk of exposure to slow-growing bacteria, especially Coxiella burnetii and Bartonella species; exposure to fastidious nonbacterial organisms; antibiotic administration preceding blood cultures; underlying valvular heart disease; right sided endocarditis; and endocarditis in patients with an intracardiac or vascular device or lines or other foreign bodies in contact with the blood.

Culture negative infective endocarditis caused by common and easily grown staphylococci or streptococci can be explained in $45 \%$ to $60 \%$ of the cases when antibiotic treatment precedes the blood cultures by 3 days or longer, as evidenced by positive polymerase chain reaction (PCR) identification of DNA from these organisms [4]. Slow growing (3-42 days) fastidious organisms (requiring specific or enriched media or cell culture systems for intracellular bacteria), such as Coxiella burnetii, Bartonella spp, Brucella spp, 
Abiotrophia spp, Listeria monocytogenes, and the HACEK group (Haemophilus sp, Actinobacilius actinomycetemcomitans, Cardiobacterium hominis, Eikenella rodens y Kingella sp), account for the remaining $40 \%$ to $55 \%$ of the cases of culture negative infective endocarditis [5]. There are a number of other uncommon bacteria that may occasionally cause endocarditis worldwide [Table 1]. However, not all of the latter would be classified as culture negative infective endocarditis according to strict criteria since many of them can be cultured using routine blood culturing methods.

It has been traditionally believed that right-sided endocarditis is more likely to be culture-negative due to bacteria being filtered by the lungs. However, there is little evidence to support this claim [6]. Recurrent bronchopneumonias are common in right-sided endocarditis [3]. Such findings in intravenous drug users or patients with intravenous catheters should suggest a diagnosis of right-sided endocarditis.

Non-infectious causes of endocarditis are classified as nonbacterial thrombotic endocarditis and are also known as marantic, Libmann-Sacks, or verrucous endocarditis. First described by Zeigler in 1888 and derived from the Greek word "marantikos", meaning "wasting away", marantic endocarditis typically involves a single valve with rare involvement of two or more valves. A review of 171 cases of thrombotic endocarditis found that $59 \%$ of the cases were in patients with underlying malignancy [7]. The vegetations were located predominantly on the mitral and aortic valves and the majority of the patients had no underlying valvular disease. Often, these are associated with an underlying hypercoagulable state. However, an undiagnosed infective endocarditis is probably the most common cause of endocarditis in cancer patients with sterile blood cultures. This may be due to 1) the increased exposure of cancer patients to broad-spectrum antimicrobial agents used as prophylaxis and preemptive or empiric therapy, 2) the small inoculum size needed to establish systemic infections in patients with severe immune dysfunction, or 3) infections due to fastidious organisms that are difficult to identify by conventional methods.

Diagnostic Workup (Table 2) 
Suspected cases of endocarditis should be investigated until all clinical, epidemiologic, echocardiographic, and laboratory data are compiled in order to be able to establish the diagnosis. In patients with infective endocarditis, the diagnosis is often definite i) when a microorganism is demonstrated by culture or histologic testing in a vegetation, an embolism, or an intracardiac abscess; ii) when active endocarditis is confirmed by histologic examination of the vegetation or intracardiac abscess; or iii) in the presence of two major clinical criteria, one major and three minor Duke criteria. The history in a patient with culture negative endocarditis may provide valuable clues to the possible etiology and can direct further investigations. A history of antibiotic therapy should be elicited as this is the most common cause of BCNE and even a short course of antibiotics can cause long lasting suppression of bacterial activity. A variety of animal exposures may predispose to certain microbiologic etiologies. Contact with sheep and cows should suggest infection with C. burnetii. The human body louse has been implicated in transmitting Bartonella Quintana. Bartonella henselae should be suspected in cat owners. Travel to the Middle East and ingesting unpasteurized milk should suggest infection with Brucella spp. Legionella should be considered in a patient with a history of recent hospitalization. Immunosuppression or prolonged antibiotic therapy should suggest endocarditis due to fungi.

The physical examination may be helpful in establishing a diagnosis of endocarditis but is unlikely to aid in defining the etiology. In a study carried out at St. Thomas' Hospital from 1975 to 2000, a total of 63 patients with BCNE were identified [8]. Of those, 17\% were afebrile; $20 \%$ had cerebral emboli; $19 \%$ had splinter hemorrhage; $17 \%$ hematuria; $15 \%$ splenomegaly; 13\% rash; $10 \%$ clubbing; $8 \%$ pulmonary emboli; 4\% peripheral emboli; 4\% subconjuctival hemorrhage; and 4\% had Osler's nodes.

\section{- Blood Culture}

Quantitative culture techniques show that blood from patients with infective endocarditis contains 1-10 bacteria per $\mathrm{ml}$ [3]. Because of the approximate correlation between the yield of bacteria from the blood and volume of blood drawn, it has been recommended that at least $10 \mathrm{ml}$ of blood be obtained for each 
culture. The use of more than three blood cultures may not improve the diagnostic yield, and therefore culture of three sets (anaerobic and aerobic) of blood drawn with an interval of at least $1 \mathrm{~h}$ within a 24-48 $\mathrm{h}$ period are normally sufficient to establish the diagnosis of culture positive endocarditis and, conversely, to indicate a possible diagnosis of BCNE. The incubation time is one of the major limiting factors for recovery of fastidious organisms, several of which may require a number of weeks to grow. If antibiotics have not been previously administered and blood cultures are negative, a fastidious growing organism should be suspected. Most organisms of the HACEK group can be isolated on enriched or chocolate agar, with the exception of Actinobacillus which may take up to 30 days to grow. Abiotrophia spp. formerly known as nutritionally deficient streptococci can be detected in routine blood culture in 2 or 3 days. However, subculture usually requires supplementation of blood agar or broth with pyridoxal hydrochloride or L-cysteine [9].

Specific media are required for some pathogens. Legionella spp. requires buffered charcoal yeast extract for optimal growth. Most Mycobacteria spp. can be isolated in standard blood culture systems but use of Middlebrook 7H13 broth should be considered especially for Mycobacterium tuberculosis [9]. Most Haemophilus spp. grow well on conventional chocolate agar, but require either exogenous hemin (X factor) or NAD (V factor) [9].

Intracellular bacteria such as Coxiella burnetii and Bartonella spp. require cultivation in cell cultures. The shell vial technique has been successfully used for isolation of Treponema whipplei and Chlamydia pneumoniae [10,11]. In patients with Bartonella spp. endocarditis the sensitivity of the shell vial assay when inoculated with blood was $28 \%$, compared with only $5 \%$ when cultured onto agar plates. The most efficient method for recovering Bartonella spp. from blood was the subculture in shell vial of the aerobic Bactec Plus (Becton Dickinson) blood culture broth on day 7 [12].

\section{- Echocardiography}


Over the past 25 years, a number of investigators have confirmed the important role of echocardiography in the diagnosis and management. Echocardiography must be performed in all patients suspected of having endocarditis. Compared to transthoracic echocardiography, transesophageal echocardiography has improved the sensitivity in defining both vegetative lesions and perivalvular infections, particularly of mural abscesses with a sensitivity of 80 to $90 \%$ [3].

\section{- Serology}

Of the fastidious organisms, serum antibody testing is available for Chlamydia spp., Legionella, Brucella, Bartonella and C. burnetii. Although, serologic testing is included as part of the Duke criteria for the diagnosis of infective endocarditis, its predictive value differs. The good predictive value of positive serology to C. burnetii has led authors to propose that a single titer of C. burnetii antibody IgG phase 1:800 be a major criterion for diagnosis $[13,14]$. For C. burnetii, the most reliable and commonly used methods are indirect immunofluorescence and complement fixation tests. Q fever endocarditis is characterized by high titers to both phase I and II antigens of C. burnetii. An IgG anti-phase-I antibody titer of 1:600 is considered to be highly predictive and sensitive, with a $98 \%$ positive predictive value [15].

Indirect and immunofluorescent assays and enzyme-linked immunosorbent assay are used in the diagnosis of Bartonella infection to detect specific antibodies. An indirect immunofluorescence assay antibody titer toward Bartonella spp. > 1:800 has a predictive value of 95\% leading to the suggestion that Bartonella serology should be included as a major criterion in the Duke criteria [16].

In a series of 10 patients reported to have Chlamydia endocarditis, eight were finally diagnosed with Bartonella endocarditis after testing their serum by cross-absorption procedures and western immunoblotting. Because of the serological cross-reaction, an elevated titer of antibody to Chlamydia spp. in a patient with BCNE should prompt Bartonella antibody testing [17]. 
Serology is a safer and effective method of diagnosing Brucella infection. At least two serological tests have to be combined to avoid false-negative results. Serum agglutination is used first for screening and complement fixation will confirm its results. A titer of 1:160 is considered positive for active infection [18]. Serologic cross-reactivity occurs between Brucella, Yersinia, and Francisella spp.

\section{- Molecular detection}

Excised valve tissue is the optimal specimen to perform 16S rDNA PCR and sequence analysis to identify the etiology, but it is available in the few patients who undergo valve resection before valve replacement. Patients who undergo surgical treatment have often been treated with antibiotics and often their blood cultures are negative at the time of surgery. Tissue samples probably contain more bacterial DNA than the equivalent volume of a blood sample, which may explain the greater sensitivity of PCR in infected valve tissues. Using 16S rDNA PCR, one study identified the etiologic agent in 9 of 12 culture negative valve tissues taken from patients who previously had positive blood cultures [4]. Two cases of BCNE were speciated by $16 \mathrm{~S}$ r DNA PCR using additional primer sets that targeted unique sequences on a second chromosomal target. In a separate study, 16S rDNA PCR provided the diagnosis and contributed to management in $21 \%(6 / 29)$ of patients with infective endocarditis (three of the six had BCNE); however, one case of culture negative endocarditis with positive histology yielded false-positive 16S rDNA PCR reactions because of specimen contamination [4].

The sensitivity of PCR and sequencing of the $16 \mathrm{~S}$ rDNA PCR gene range from $41 \%$ to $96 \%$ and specificity from $95 \%$ to $100 \%$ whereas sensitivity and specificity of valve culture ranged from $8 \%$ to $26 \%$ and $56 \%$ to $94 \%$, respectively $[19,20]$. Molecular analysis has higher sensitivity than blood and tissue culture and can detect causative organisms even in patients receiving prior antibiotic administration or having organisms that are difficult to culture.

Universally, the biggest asset of 16S RNA gene PCR is also its biggest drawback. Contamination of PCR reactions with background bacterial DNA has been a major limitation even in the face of rigorous 
techniques intended to prevent specimen contamination. Although, the sensitivity and specificity of PCR is well established in resected valves, the usefulness of this technique in blood is still debated. The low sensitivity of PCR in blood is likely due to pre-PCR contamination. New and ingenious strategies are required to improve the sensitivity and specificity of $16 \mathrm{~S}$ rDNA PCR reactions and to identify patients with BCNE. These strategies should include further reduction of background bacterial DNA, cleaner techniques for sample acquisition; and the application of these techniques to blood, the most relevant specimen in infective endocarditis.

\section{- Histology}

Because histopathology can confirm the diagnosis by revealing valvular inflammation, the vegetation, the organisms or other changes consistent with endocarditis, the histology of the resected valve remains the gold standard for the diagnosis and is a major criterion of the Duke classification.

A number of different stains can be used to help identify various organisms implicated in IE as well as confirm the presence of inflammation (Table 3). A variety of specific stains can also be used based on clinical indications. If the patient has risk factors for a Mycobacterial infection, valves should be stained with Ziehl-Nielsen staining for acid-fast bacteria. The Gimenez stain allows detection of C. burnetii and Legionella species. The Kinyoun stain can also detect mycobacterial species. It also stains large macrophages containing dark red granules seen in Chlamydia endocarditis. For detection of fungi, the Gomori-Grocott's silver stain provides the best contrast.

\section{- Detection of autoantibodies}

Rheumatoid factor, antinuclear antibodies, and anti-DNA antibodies need to be checked in all patients with BCNE to rule out non-infectious causes. In a prospective study of 759 cases in France, 19 (2.5\%) were classified as having non-infective endocarditis [21]. Among those, histopathology identified marantic endocarditis, Libmann-Sacks endocarditis, and Behcet disease in 7, 4, and 1 cases, respectively. Subsequently, by evaluating presence of antinuclear antibodies in 129 of 290 patients for whom results of 
all assays were negative and by calling their physicians in charge, 7 additional patients were identified in whom diagnosis of autoimmune disease had been done elsewhere using diagnostic criteria, including 5 patients with Libmann-Sacks endocarditis and 2 with rheumatic arthritis.

These data demonstrate that the BCNE picture may include cases of nonbacterial thrombotic endocarditis associated with cancers and autoimmune diseases. The results support the usefulness of a systemic detection of rheumatoid factor and antinuclear antibodies, as well as histopathologic analysis of all valve tissue taken from patients with suspected endocarditis.

\section{- Other studies}

Patients with BCNE are usually afebrile as the process often appears to indicate prolonged and unchecked stimulation of the immune system. They often present with symptoms of heart failure. Signs and symptoms secondary to emboli include acute meningitis with sterile spinal fluid, hemiplegia in the distribution of the middle cerebral artery, regional infarcts that cause painless hematuria, infarction of the kidney or spleen, unilateral blindness caused by occlusion of a retinal artery, and myocardial infarction arising from embolization of a coronary artery. Emboli of right sided endocarditis commonly produce pulmonary infarcts. The rate of embolization is related to the organism, the size of the vegetation and its rate of growth or resolution, and its location. Deposition of circulating immune complexes in the kidney may produce interstitial nephritis or proliferative glomerulonephritis leading to renal failure. Similarly, various musculoskeletal symptoms arise from immunologically mediated synovitis. In $50 \%$ of patients with cerebral emboli, the event is the first manifestation of endocarditis and is associated with a 2 to 4 times higher mortality rate. Stroke in younger people should always raise the possibility of underlying infective endocarditis. Radionuclide scans of the spleen are useful to help rule out a splenic abscess, which is a cause of bacteremia that is refractory to antibiotic therapy. A computed tomography scan of the head should be obtained in patients who exhibit central nervous system symptoms [22].

\section{Treatment of BCNE}




\section{Medical Treatment of Culture Negative Infective Endocarditis}

There are a number of published recommendations for empiric antibiotic treatment of BCNE [23-26]. Rational empiric treatment should include an antibiotic that is active against the bacterial cell wall and an aminoglycoside. The American Heart Association/American College of Cardiology guidelines recommend treatment with ampicillin and gentamycin, or vancomycin and gentamycin, plus ciprofloxacin [24]. The European Society of Cardiology recommends vancomycin plus gentamycin [25]. In Germany, current guidelines recommend ampicillin and gentamycin plus ceftriaxone [26]. If a patient has had significant exposure to farm animals, treatment with ciprofloxacin in combination with rifampin or doxycycline can be initiated to cover for Bartonella or Coxiella infection. If the patient continues to deteriorate despite initiation of empiric therapy, treatment for HACEK, Abiotrophia, and Bartonella can be initiated with ceftriaxone and gentamycin.

The International Collaboration on Endocarditis is a comprehensive registry with epidemiological data on more than 5000 endocarditis patients from 63 centers around the world [27]. Among 1779 patients enrolled between 2000 and 2003 from the U.S., Brazil, Australia, New Zealand, Europe, and the Middle East, the most common causative pathogen was S. aureus (558 cases, 31\%). However, in a nationwide surveillance conducted in Japan between 2000-2001, S. aureus was still the second most common cause (17\%) after Streptococcus viridans (32\%). Taking into account that one of the primary reasons for culture negative infective endocarditis is pre-treatment with antibiotics, it is feasible that there exists a large proportion of unrecognized staphylococci infection.

There are also concerns regarding the use of vancomycin. Firstly, vancomycin has poor tissue penetration and secondly, there is the continuing debate over bactericidal versus bacteriostatic action of antibiotics. Clinical studies comparing vancomycin with beta-lactam for example have shown a significantly worse outcome with vancomycin in terms of cure rates (62\% vs. 84\%) [28]. Vancomycin may still be needed to treat patients infected with methicillin resistant staphylococcus aureus (MRSA) which has been on the 
rise and soon it may be necessary to find suitable alternative agents to vancomycin. A systematic review from 1995 to 2006 was performed to assess the effectiveness and safety of linezolid for treatment [29]. Although there are no randomized controlled trials, case series and case reports have demonstrated that linezolid should be considered in patients with limited options [29].

A randomized controlled trial of 124 patients with S. aureus bacteremia with or without endocarditis (mainly right-sided) was conducted to compare the efficacy of daptomycin with vancomycin plus gentamycin in cases of MRSA or anti-staphylococcal penicillin plus gentamycin in methicillin sensitive staphylococcus aureus cases [30]. In that study, there was a strong trend towards superiority for daptomycin over vancomycin/gentamycin, indicating that this new agent may be a suitable substitute for vancomycin for management of MRSA bacteremia [30].

The issue of monotherapy with gentamycin versus combination therapy for S. aureus endocarditis has been analyzed in two previously published meta-analyses [31, 32]. A large meta-analysis by the Cochrane Database Service included 7500 sepsis patients from 64 studies and found that combination therapy with gentamycin was not superior to monotherapy with beta-lactam antibiotics [31]. A separate meta-analysis by Falagas examined the subset of infective endocarditis studies, covering streptococci and staphylococci pathogens [32]. The results demonstrated that neither for death nor clinical cure as an endpoint, was there a real benefit for combination therapy with gentamycin.

In certain patients with $\mathrm{BCNE}$, situations may arise that deserve special consideration because specific therapeutic regimens are to be used. For Coxiella burnetti, doxycycline, rifampicin and fluoroquinolones are efficacious in vitro but none of these is bactericidal. The duration of treatement is very long, often several years [32]. Serology appears to be the most reliable criterion of cure, when phase I IgG titers are $<200$ and phase I IgA titers are undetectable. Doxycycline is the cornerstone antibiotic, and its efficacy is improved when combined with hydrochloroquine which acts as a lysomotropic alkalinizing agent. This combination helps decrease the duration of antibiotic treatment and the failure and relapse rates [33]. 
Bartonella spp. has in vitro susceptibility to beta-lactam agents, aminoglycosides, macrolides, tetracyclines, and rifampicin. A standard antibiotic regimen has not been definitely established, but retrospective data support a combination of gentamycin for two weeks and doxycycline or ceftriaxone for four to six weeks. A large proportion of patients require valve surgery due to the destructive nature of both B. henselae and B. quintana. In one series, valve replacement was performed in $80 \%$ of cases of Bartonella endocarditis [34]. In that series, the mortality rate in 101 patients with Bartonella endocarditis was $12 \%$. Improved survival was associated with aminoglycoside therapy.

HACEK-associated endocarditis has a favorable outcome in $85-90 \%$ of patients who are treated either medically or in combination with valve replacement [35]. The standard recommended treatment should include a beta-lactamase stable cephalosporin such as a ceftriaxone.

Surgical treatment in combination with medical therapy is necessary in the majority of patients with Brucella endocarditis. In a series by Requera, $72 \%$ of 11 patients required valve replacement [36]. Survival in that series was $91 \%$. Standard therapy should include a combination of doxycycline and rifampicin or streptomycin for a minimum of three months. If valve replacement is undertaken, antimicrobial therapy should continue for six to eight weeks postoperatively. Antibody titers can be used to monitor response to treatment.

Fungal endocarditis treatment, in most instances, should include both medical and surgical therapy. Amphotericin B should be the drug of choice until susceptibility testing can be completed. Other options include the addition of flucytosine to amphotericin B, or fluconazole. Newer agents such as voriconazole have not been well studied. Generally, patients may require greater than six months of therapy and may need lifelong suppressive therapy. Even with optimal surgical and medical management, the prognosis for patients with fungal endocarditis has been poor compared with endocarditis caused by other pathogens. In the two largest series reported in the literature, the mortality rates were $56 \%$ and $77 \%$, respectively [3739]. 
Endocarditis caused by Legionella is rare and occurs mainly in patients with prosthetic valves. The usual treatment regimen consists of a combination of a fluoroquinolone and rifampin. The duration of treatment should be at least two months, and valve surgery is almost always required.

Treatment of T. whipplei endocarditis has not been standardized. Most patients with Whipple's disease are treated with cotrimoxazole, ceftriaxone, or doxycycline for a minimum of six weeks and more frequently for six months to a year. The prognosis is a yet unknown [10].

\section{Medical treatment of Non Bacterial Thrombotic Endocarditis}

Choosing the appropriate therapy in patients with nonbacterial thrombotic endocarditis is a major dilemma. Despite absence of available data in the literature, thrombotic lesions may become secondarily infected by circulating bacteria and preventive antibiotic therapy before procedures known to produce bacteremia may be indicated [40]. Long-term anticoagulant treatment may be necessary in patients with primary antiphospholipid syndrome and those with a past history of thromboembolic events.

Thrombotic endocarditis has often been a precipitating event in a patient with disseminated intravascular coagulopathy [41]. Thus, the underlying process which initiates and sustains the coagulation abnormalities in patients with thrombotic endocarditis should be treated. In situations where the underlying process cannot be controlled rapidly and significant thrombosis, hemorrhage, or embolization may occur efforts must be directed at controlling the pathologically altered coagulation mechanism. Heparin has been noted to prevent deposition of fibrin and platelets on the heart valves. The long-term use of heparin has been recommended in patients with Trousseau's syndrome and little benefit was observed with warfarin therapy. Bell et al described two patients with Trousseau's syndrome, who experienced frequent arterial and venous thrombotic events that resulted in sequential amputations [42]. Warfarin therapy was not beneficial in preventing the occurrence of these events. However, intravenous heparin prevented the thrombotic events. 


\section{Surgical Treatment}

Valve replacement remains an important management option for patients with BCNE. An analysis using propensity score matching in a cohort from the International Collaboration on Endocarditis showed that surgery was the only overall protective factor against in-hospital mortality in proven cases of endocarditis [43]. Valve replacement is indicated in approximately half of the patients because of severe complications. Reasons to consider early surgery in the active phase, while the patient is still receiving antibiotic treatment, are to avoid progressive heart failure and irreversible structural damage caused by severe infection and to prevent systemic embolism. On the other hand, surgical therapy during the active phase of the disease is associated with significant risk. Surgery is justified in patients with high-risk features which make the possibility of cure with antibiotic treatment unlikely and who do not have comorbid conditions or complications that make the prospect of recovery remote.

It is advisable that each case must be individualized and all factors associated with increased risk identified at the time of the diagnosis. Frequently, the need for surgery will be determined by a combination of several high-risk features. In some cases, surgery needs to be performed on an emergency (within 24 hours) or urgent (within a few days) basis, irrespective of the duration of antibiotic treatment. In other cases, surgery can be postponed to allow 1 or 2 weeks of antibiotic treatment under careful clinical and echocardiographic observation. The three main indications for early surgery in infective endocarditis are heart failure, uncontrolled infection, and prevention of embolic events. In a recent study, Kang et al conducted a randomized trial comparing early valve surgery with conventional treatment in patients with infective endocarditis and showed that early surgery performed within 48 hours after diagnosis reduced the composite primary end point of death from any cause or embolic events by effectively reducing the risk of systemic embolism. Moreover, these improvements in clinical outcomes were achieved without an increase in operative mortality or recurrence of infective endocarditis [44]. 
Heart failure is the most frequent complication of endocarditis. Unless severe co-morbidity exists, the presence of heart failure indicates early surgery in patients with native valve endocarditis. Uncontrolled infection is most frequently related to perivalvular extension or "difficult-to-treat" organisms. Thus, the presence of locally uncontrolled infection indicates early surgery in patients with native valve endocarditis. Embolism is very frequent, complicating $20 \%-50 \%$ of cases of infective endocarditis, falling to $6 \%-21 \%$ after initiation of antibiotic therapy [45].

The risk of embolism is highest during the first 2 weeks of antibiotic therapy and is clearly related to the size and mobility of the vegetation. Risk is increased with large (>10 $\mathrm{mm})$ vegetations and is particularly high with very mobile and larger $(>15 \mathrm{~mm})$ vegetations. The highest incidence of embolic complications is seen with aortic and mitral valve infections and with S. aureus, Candida and HACEK group bacteria and Abiotrophia organisms. The decision to operate on early to prevent embolism is always difficult and specific for the individual patient. Governing factors include size and mobility of the vegetation, previous embolism, type of microorganism, and duration of antibiotic therapy.

The indications and appropriate timing for surgery in noninfective thrombotic endocarditis have not been formally studied and should be individualized. Severe valvular dysfunction and recurrent embolic events despite anticoagulation are clear surgical indications. In addition, persistent doubts about the possible infective nature of the vegetation may favor surgical removal of the lesion [46]. If the diagnosis is strongly suspected, preservation of the affected valve may be possible in selected cases in which the valve is found to be structurally and functionally normal after the vegetations are removed. This is in contrast to bacterial endocarditis, in which the need for removing thoroughly all infected tissue in the acute phase nearly always mandates excision of the valve and prosthetic replacement. Repair of the valve in some instances might be indicated in patients with serial negative blood cultures and especially in patients with non-infective endocarditis due to other etiologies (figure 1). 


\section{$\underline{\text { Conclusions }}$}

BCNE remains a formidable clinical challenge. To date, definitive studies of infective endocarditis have been difficult to perform because of its heterogeneous nature. Launched in 1999, the International Collaboration on Endocarditis was conceived to develop a large global database of patients whose clinical, echocardiographic, and microbiological findings have been characterized using standard methodology. This resource offers the opportunity for major advances in the understanding and treatment of infective endocarditis over the next two decades.

Increased emphasis on symptoms and signs coupled with improved likelihood of identification of a causative pathogen using serology, additional culture, or newer histological and molecular techniques will improve the sensitivity of the diagnosis and increase the chance of successful therapeutic intervention. These measures, combined with close collaboration and communication between the cardiologist, cardiac surgeon, and microbiologists, are essential to ensure optimal diagnosis and management and a favorable outcome. 


\section{$\underline{\text { References }}$}

1. Lepidi H, Durack DT, Raoult D: Diagnostic methods, current best practices and guidelines for histologic evaluation in infective endocarditis. Infect Dis Clinics North Am. 2002; 16: 339-361.

2. Durack DT, Lukes AS, Bright DK: New criteria for diagnosis of infective endocarditis; utilization of specific echocardiographic findings. Am J Med 1994; 96: 200-209.

3. Brouqui P, Raoult D: Endocarditis due to rare and fastidious bacteria. Clin Microbiol Rev 2001; $14: 177-207$.

4. Madico GE, Rice PA: 16s-Ribosomal DNA to Diagnose Culrure-Negative Endocarditis. Current Infectious Disease Reports 2008; 10: 280-286.

5. Fournier PE, Lelievre H, Eykyn SJ, et al: Epidemiologic and clinical characteristics of Bartonella quintana and Bartonella henselae endocarditis: a study of 48 patients. Medicine (Baltimore) 2001; 80: $245-51$.

6. Houpikian P, Raoult D: Blood culture-negative endocarditis in a reference center: etiologic diagnosis of 348 cases. Medicine (Baltimore) 2005; 84: 162-73.

7. Steiner I: Nonbacterial thrombotic endocarditis- a study of 171 case reports. Ceskoslovenska Patologie 1993; 29: 58-60.

8. Lamas CC, Eykyn SJ: Blood culture negative endocarditis: analysis of 63 cases presenting over 25 years. Heart 2003; 89: 258-262.

9. Koegelenberg CF, Doubell AF, Orth H, Reuter H: Infective endocarditis in the Western Cape Province of South Africa: a three-year propective study. QJM 2003; 96: 217-225.

10. Fenollar F, Leipidi H, Raoult D: Whipple's endocarditis: review of the literature and comparison with Q fever Bartonella infection and blood culture positive endocarditis. Clin Infect Dis 2001; 33: 1309-1316. 
11. Gdoura R, Pereyre S, Frikha I, et al: Culture-negative endocarditis due to Chlamydia pneumonia. J Clin Microbiol 2002; 40: 718-720.

12. La Scola B, Raoult D: Culture of Bartonella Quintana and Bartonella henselae from human samples: a 5 -year experience (1993 to 1998). J Clin Microbiol 1999; 34: 2270-2274.

13. Fournier PE, Casalta JP, Habib G, et al: Modification of the diagnostic criteria proposed by the Duke Endocarditis Service to permit improved diagnosis of Q fever endocarditis. Am J Med 1996; 100: 629633.

14. Li JS, Sexton DJ, Mick N, et al: Proposed modifications to the Duke criteria for the diagnosis of infective endocarditis. Clin Infect Dis 2000; 30: 633-638.

15. Dupont HT, Thirion X, Raoult D: Q fever serology: cutoff determination for microimmunofluorescence. Clin Diagn Lab Immunol 1994; 1:189-196.

16. Fournier PE, Mairandi JL, Raoult D: Value of microimmunofluorescence for diagnosis and followup of Bartonella endocarditis. Clin Diagn Lab Immunol 2002; 9: 795-801.

17. Houpikian P, Raoult D: Western immunoblotting for Bartonella endocarditis. Clin Diagn Lab Immunol 2003; 10: 95-102.

18. Young E: Serologic diagnosis of human brucellosis: analysis of 214 cases by agglutination tests and review of the literature. Rev Infect Dis 1991; 13: 359-372.

19. Miyazato A, Ohkusu K, Tabata M, et al: Comparative molecular and microbiological diagnosis of 19 infective endocarditis cases in which causative microbes were identified by PCR-based DNA sequencing from the excised heart valves. J infect Chemother 2012;18: 318-23. 
20. Goldenberger D, Kunzil A, Vogt P, Zbinden R, Altwegg M: Molecular diagnosis of bacterial endocarditis by broad-range PCR amplification and direct sequencing. J Clin Microbiol 1997; 35: 2733 2739.

21. Pierre-Edouard Fournier, Franck Thuny, Hervé Richet, Hubert Lepidi, Jean-Paul Casalta, Jean-Pierre Arzouni, Max Maurin, Marie Célard, Jean-Luc Mainardi, Thierry Caus, Frédéric Collart, Gilbert Habib and Didier Raoult. Comprehensive Diagnostic Strategy for Blood Culture-Negative Endocarditis: A Prospective Study of 819 New Cases. Clin. Infectious Diseases 2010; 51(2):131-140.

22. Cheitlin MD, Armstrong WF, Aurigemma GP, et al: ACC/AHA/ASE 2003 guideline update for the clinical application of echocardiography: summary article: a report of the American College of Cardiology/American Heart Association Task Force on Practice Guidelines (ACC/AHA/ASE Committee to Update the 1997 Guidelines for the Clinical Application of Echocardiography). Circulation 2003; 108 : $1146-62$

23. Naber CK, Erbel R: Infective endocarditis with negative blood cultures. Int J Antimicrobial Agents 2007; S32-S36

24. Baddour LM, Wilson WR, Bayer AS, et al: Infective endocarditis: diagnosis, antimicrobial therapy, and management of complications. Circulation 2005; 111: e394-434.

25. Horstkotte D, Follath F, Gutschik E, et al: Guidelines on prevention, diagnosis and treatment of infective endocarditis executive summary; the task force on infective endocarditis of the European society of cardiology. Eur Heart J 2004; 25: 267-76.

26. Naber CK, Bauhofer A, Block M, et al: S2 Guideline for diagnosis and therapy of infectious endocarditis. Z. Kardiol 2004; 93: 1005-21.

27. Fowel Jr VG, Miro JM, Hoen B, et al: ICE Investigators. Staphylococcus aureus endocarditis: a consequence of medical progress. JAMA 2005; 293: 3012-21. 
28. Fowel Jr VG, Sanders LL, Sexton DJ, et al: Outcome of Staphylococcus aureus bacteremia according to compliance with recommendations of infectious diseases specialists: experience with 244 patients. Clin Infect Dis 1998; 27: 478-86.

29. Falagas ME, Manta KG, Ntziora F, Vardakas KZ: Linezolid for the treatment of patients with endocarditis: a systemic review of the published evidence. J. Antimicrob Chemother 2006; 58: 273-80.

30. Fowel Jr VG, Boucher HW, Corey GR, et al: Daptomycin versus standard therapy for bacteremia and endocarditis caused by Staphylococcus aureus. N Engl J Med 2006; 355: 653-65.

31. Paul M, Silbiger I, Grozinsky S, Soares-Weiser K, Leibovic L: Beta lactam antibiotic monotherapy versus beta lactam-aminoglycoside antibiotic combination therapy for sepsis. Cochrane Database Syst Rev 2006; Jan 25(1): CDC003344.

32. Falagas ME, Matthaiou DK, Bliziotis IA: The role of aminoglycosides in combination with a betalactam for the treatment of bacterial endocarditis: a meta-analysis of comparative trials. J. Antimicrob Chemother 2006; 57: 639-47.

33. Hoen B: Epidemiology and Antibiotic treatment of infective endocarditis: An update. Heart 2006; 92 : $1694-1700$.

34. Raoult D, Fournier PE, Vandenesch F, et al. Outcome and treatment of Bartonella endocarditis. Arch Intern Med 2003; 163: 226-230.

35. Das M, Badley AD, Cockerill FR, Steckelberg JM, Wilson WR: Infective endocarditis caused by HACEK microorganisms. Ann Rev of Med 1997; 48: 25-33.

36. Requera JM, Alarcon A, Miralles F, Juarez C, Colmenero JD: Brucella endocarditis: Clinical diagnostic and therapeutic approach. Eur J of Clin Microbial Infect Dis 2003; 22: 647-650. 
37. Ellis ME, Al-Abdely H, Sandridge A, Ventura W: Fungal endocarditis: Evidence in the world literature, 1965-1995. Clin Infect Dis 2001; 32: 50-62.

38. Pierrotti LC, Baddour LM: Fungal endocarditis, 1995-2000. Chest 2002; 122: 302-310.

39. Thuny F., Fournier PE, Casalta JP, et al: Investigation of blood culture-negative early prosthetic valve endocarditis reveals high prevalence of fungi. Heart 2010; 96: 743-747.

40. Reisner SA, Brenner B, Haim N, Edoute Y, Markiewicz W: Echocardiography in nonbacterial thrombotic endocarditis: from autopsy to clinical entity. J Am Soc Echocardiogr. 2000; 13: 876-81.

41. Lopez JA, Ross RS, Fishbein MC, Siegel RJ: Nonbacterial thrombotic endocarditis: a review. Am Heart J. 1987; 113: 773-84.

42. Bell WR, Starksen NF, Tong S, Porterfield JK: Trousseau's syndrome. Devastating coagulopathy in the absence of heparin. Am J Med 1985; 79: 423-430.

43. Aksoy O, Sexton DJ, Wang A, et al: Early surgery in patients with infective endocarditis: a propensity score analysis. Clin Infect Dis. 2007; 44: 364-72.

44. Kang DH, Kim YJ, Kim SH et al: Early Surgery versus Conventional Treatment for Infective Endocarditis. N Engl J Med 2012; 366:2466-2473.

45. Bayer AS, Bolger AF, Taubert KA, et al: Diagnosis and management of infective endocarditis and its complications. Circulation 1998; 98: 2936-2948.

46. Rabinstein AA, Giovanelli C, Ricci M, Romano JG, Koch S, Forteza AM: Surgical treatment of nonbacterial thrombotic endocarditis presenting with stroke. J Neurol 2005; 252: 352-355. 
Table 1. Causative Organisms in Blood Culture Negative Infective Endocarditis (BCNIE) Caused by other than Common Bacterial Agents.

\begin{tabular}{|l|}
\hline Microorganism \\
\hline Coxiella burnetii \\
\hline Bartonella spp. \\
\hline Brucella spp. \\
\hline Abiotrophia spp. \\
\hline Actinobacillus \\
\hline Haemophilus aphrophilus \\
\hline Cardiobacterium hominis \\
\hline Corynobacterium diptheriae \\
Haemophilus parainfluenzae \\
Listeria monocytogenes \\
\hline Erysipelothrix rhusiopathiae \\
\hline Neisseria spp. \\
\hline Gamella spp. \\
\hline Mycoplasma spp. \\
\hline Campylobacter spp. \\
\hline Pasteurella \\
\hline Mycobacterium spp. \\
\hline Legionella spp. \\
\hline Whipple's disease bacillus \\
\hline Francisella tularensis \\
\hline
\end{tabular}


Table 2. Common Diagnostic Tests Utilized for the Diagnosis of Blood Culture Negative Endocarditis. [PCR: Poylmerase chain reaction; RF: Rhematoid factor; ANA: antinuclear antibody].

Blood Cultures

Echocardiography

Transthoracic

Transesophageal

Serology

Immunoglobulin assay

Immunofluorescent assay

Molecular Detection

16 rDNA PCR

Histolopathology

Tissue stains

Detection of antibodies

$R F$

ANA

anti-DNA

Computerized tomographic (CT) scans

Head, chest, abdomen

Radionuclide scans of abdomen

Bone scan 
Table 3. Common Histological Stains Used in the Diagnosis of Blood Culture Negative Infective Endocarditis (BCNIE).

\begin{tabular}{|l|l|}
\hline Tissue stain & Microorganism \\
\hline & \\
Brown-Hoops gram stain & Gram positive bacteria \\
Brown-Brenn gram stain & Gram negative bacteria \\
Periodic acid-Schiff & Tropheryma whipplei, fungi \\
Warthin-Starry silver stain & Bartonella species \\
Ziehl-Nielsen & Acid-fast bacilli \\
Gimenez & Coxiella burnetti, Legionella species \\
Kinyoun, Mchiavello & Chlamydia species, Mycobacteria, \\
Gomori-Grocolt silver stain & Fungi \\
Acridine orange & Any bacterium, Mycoplasma spp, Fungi \\
Giemsa & Any bacterium \\
& \\
\hline
\end{tabular}

